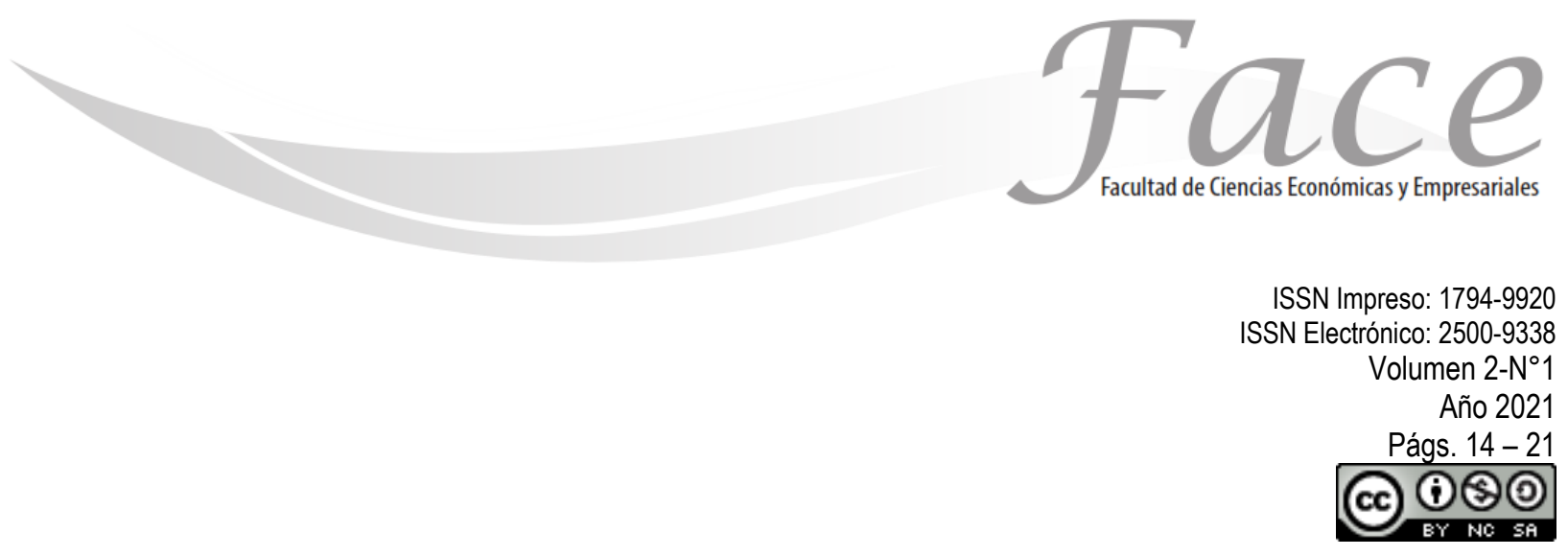

\title{
LA PUBLICIDAD DIGITAL Y EL DESAFIO DE LA SATURACIÓN PUBLICITARIA PARA EL EMPRESARIO
}

\author{
Roy Priego Reyes \\ Enlace ORCID: $\underline{0000-0002-1819-2384}$ \\ Germán Martínez Prats \\ Enlace ORCID: 0000-0001-6371-448X \\ Mariela Adriana Rodríguez Ocaña \\ Enlace ORCID: $\underline{0000-0002-3110-7439}$
}

Fecha de Recepción: Febrero 5

Fecha de Aprobación: Mayo 20

\section{Resumen:}

Ya es una realidad que el internet han desplazado a los medios de comunicación tradicionales. Su rápida evolución, ha modificado la forma en la que el ser humano se comunica e interactúa con el mundo, Noguez (2016). De igual forma, ha afectado campos tan diversos como la medicina, la economía, la política, la publicidad, etc. Precisamente, la unión entre publicidad y el mundo digital ha derivado en anuncios más interactivos, diversos y especializados, su eficacia como medio, ha generado que las inversiones en publicidad digital crezcan exponencialmente en los últimos años.

Sin embargo, existe una problemática que ya afectaba a los medios analógicos y continua en el medio digital. La saturación publicitaria, puede definirse como: Un exceso de mensajes publicitarios en un medio determinado, lo cual genera indiferencia en los consumidores y por consiguiente la perdida de efectividad de esta.

El presente artículo, tiene como objetivo analizar por medio de una revisión bibliográfica cual es el impacto que ha tenido la saturación publicitaria en la audiencia presente en internet. Lo anterior con el fin de determinar sus efectos en la efectividad de la publicidad.

Palabras clave: Publicidad digital, saturación publicitaria, internet.

* Maestría en mercadotecnia y gerencia de marca, Profesor Universidad Montemorelos- México. Contacto: roypr33@hotmail.com.

**Doctor en Gobierno y administración pública, Profesor investigador Universidad Juárez Autónoma de Tabasco-DACEA- México. Contacto: germanmtzprats@hotmail.com

*** Doctora en Administración, Profesor Universidad Juárez Autónoma de Tabasco -DACEA. México. Contacto: dacea_academia@hotmail.com 


\title{
DIGITAL ADVERTISING AND THE CHALLENGE OF ADVERTISING SATURATION FOR THE ENTREPRENEUR
}

\author{
Abstract:
}

It is already a reality that the internet has displaced the traditional media. Its rapid evolution has modified the way in which the human being communicates and interacts with the world, Noguez (2016). In the same way, it has affected fields as diverse as medicine, economics, politics, advertising, etc. Precisely, the union between advertising and the digital world has resulted in more interactive, diverse and specialized advertisements; its effectiveness as a medium has led to investments in digital advertising growing exponentially in recent years.

However, there is a problem that already affected analog media and continues in digital media; Advertising saturation can be defined as: An excess of advertising messages in a given medium, which generates indifference in consumers and consequently the loss of effectiveness of this.

The objective of this article is to analyze by means of a bibliographic review what is the impact that advertising saturation has had on the audience present on the internet. The foregoing in order to determine its effects on the effectiveness of advertising.

Keywords: Digital advertising, advertising saturation, internet.

\section{A PUBLICIDADE DIGITAL E O DESAFIO DA SATURAÇÃO DA PUBLICIDADE PARA O EMPREENDEDOR}

\section{Resumo:}

Já é uma realidade que a internet substituiu os meios de comunicação tradicionais. Sua rápida evolução modificou a forma como o ser humano se comunica e interage com o mundo, Noguez (2016). Da mesma forma, afetou campos tão diversos como medicina, economia, política, publicidade, etc. Precisamente, a união da publicidade com o mundo digital resultou em anúncios mais interativos, diversificados e especializados, sua eficácia como meio fez com que os investimentos em publicidade digital crescessem exponencialmente nos últimos anos.

No entanto, existe um problema que já afetou a mídia analógica e continua na mídia digital; A saturação publicitária pode ser definida como: Excesso de mensagens publicitárias em um determinado meio, o que gera indiferença nos consumidores e consequentemente a perda de eficácia deste.

O objetivo deste artigo é analisar, por meio de uma revisão bibliográfica, qual o impacto que a saturação publicitária teve sobre o público presente na internet. $\mathrm{O}$ anterior, a fim de determinar seus efeitos sobre a eficácia da publicidade.

Palavras-chave: Publicidade digital, saturação publicitária, internet. 


\section{INTRODUCCIÓN:}

La publicidad ha crecido de manera exponencial en todo el mundo. Las inversiones en publicidad aumentan año con año, llegando a cifras descomunales, solo en el año 2017 se invertía la cantidad de 137,530,000 millones de dólares Eguizábal (2018). La lucha por sobrevivir en un mercado saturado de marcas necesita de una constante evolución e inversión en mercadotecnia.

Por lo tanto, surge una situación alarmante para las empresas modernas. Javier Regueira, socio fundador de Pop Up Brand Content, anunciaba en Marketing News que la publicidad tradicional parece herida de muerte. 9 de cada 10 consumidores declaran estar en contra de los anuncios y solo se recuerda un $3 \%$ de los 5,000 que el usuario promedio recibe durante el día. Lo anterior se traduce en el hecho de que, por cada 5,000 impactos publicitarios, tan solo recordamos 150 anuncios Mord y Gilson (1985). En el transcurso de un año, recibimos 54,750 impactos, logrando recordar un total 4,500 al mes.

Una encuesta sobre los problemas que afectan a los internautas en la red fue realizada por la Asociación para la Investigación de Medios de Comunicación (AIMC). Y esta revelo que un 59\% de los encuestados eligen "demasiada publicidad" como el principal problema del internet moderno.

Queda en evidencia que el verdadero problema no es nuestra capacidad para recordar si no en la descomunal cantidad de anuncios con los que tomamos contacto en nuestro diario vivir.

Hammer, Riebe y Kennedy (2009) afirman que la saturación publicitaria puede definirse como el exceso de publicidad en un determinado medio de comunicación, se caracteriza por generar rechazo e indiferencia en el público por lo que perjudica la efectividad de la publicidad y por consiguiente en las ventas de una empresa.

Aunque anteriormente se han hecho estudios sobre este problema lo cierto es que los medios tradicionales como la radio y la televisión han sido lentamente remplazados por uno de los mayores avances tecnológicos de nuestros días, el internet. Es precisamente la rápida aceptación de este servicio lo que ha impactado en el comportamiento del consumidor, así como en la mezcla de marketing de las empresas, Noguez (2016).

El presente artículo, tiene como objetivo analizar el impacto que ha tenido la saturación publicitaria en la audiencia presente en los medios de comunicación digitales. Para este propósito, se toman en cuenta tres factores determinan la efectividad de la publicidad, lo cuales son: la percepción de la audiencia, la recordación de anuncios y la recordación de marca.

\section{MARCO TEÓRICO}

La saturación publicitaria es un problema relativamente nuevo que surge a finales del siglo $X X$ y que ha cobrado mayor importancia en la actualidad, debido al incremento de los competidores. Analizando la evolución de la mercadotecnia, podemos encontrar el nacimiento de la publicidad moderna en los orígenes de la imprenta en el año de 1450, la rápida aceptación de esta nueva tecnología fue el punto de partida para que los comerciantes comenzaran a promocionar sus negocios por medio de rudimentarias impresiones con muy baja definición. Tiempo después, por medio de periódicos, revistas y libros.

El 12 de diciembre de 1901 el italiano Guillermo Marconi inventa la radio, un nuevo medio de comunicación capaz de transmitir el sonido hasta los hogares de los consumidores, la publicidad se adapta a este nuevo descubrimiento por medio de spots publicitarios y programas de radio.

En 1959 surge una nueva tecnología que abre las puertas a nuevas posibilidades, la televisión, aparece en escena y cambia nuevamente las reglas del juego, debido a la capacidad de este medio para transmitir un mensaje por medio de imágenes en movimiento y sonidos. Eguizábal (2008)

Es importante mencionar que durante este breve análisis de la evolución el termino: "saturación publicitaria" no existía como tal debido a las características de los medios y al equilibrado número de competidores en el mercado, durante la revolución industrial y la época de la segunda guerra mundial, lo más importante era la producción a gran escala y la eficacia en el proceso, las empresas no se preocupaban por agradar a sus clientes, por lo que la publicidad era relativamente sencilla. Eguizábal (2008) Sin embargo, los autores Webb y Ray (1979) comienzan los primeros estudios serios sobre la recordación de marca, en los que llegan a dos conclusiones importantes, en primer lugar, que las personas son capaces de recordar un número limitado de anuncios y marcas, por lo tanto, un exceso de estos trae como consecuencia que el usuario no logre recordar toda la publicidad a la que es expuesto. Por otro lado, también lograron determinar que existe una 
Roy Priego Reyes - Germán Martínez Prats - Mariela Adriana Rodríguez Ocaña

correlación entre la posición que ocupan los anuncios en un bloque publicitario y su efectividad para ser recordado por el público, mientras que los primeros anuncios son mayormente recordados (efecto Primacy) los anuncios finales tienden a generar indiferencia y por consiguiente a ser menos efectivos (efecto recency).

Mord y Gilson (1985) realizaron un estudio similar y determinaron que la efectividad de los anuncios expresado en persuasión y agrado, se veía seriamente afectada de manera proporcional a la cantidad de saturación publicitaria en el medio de comunicación, sin embargo, debido a la naturaleza del estudio en la que se forzaba a los participantes a estar expuestos a la publicidad, los niveles de atención no se vieron afectados. Mord y Gilson (1985),

En 1993 Brown y Rothschild encontraron que el incremento en la saturación publicitaria podía perjudicar la efectividad en los anuncios ya que influía de manera negativa en la recordación de marcas específicas, los resultados arrojaron las siguientes proporciones:

De 26 anuncios la recordación de marca estuvo en un nivel del $74 \%$ considerado un nivel de saturación publicitaria bajo. En 29 anuncios se logró una recordación de marca del $71 \%$, considerado un nivel de saturación intermedio. Finalmente, la exposición a 34 anuncios dio una recordación de marca de 68\% para un nivel alto de saturación. Después de analizar los resultados se concluyó que existe una relación proporcional en la que la recordación de marca se ve afectada por un incremento en el número de anuncios publicitarios. Brown y Rottshield (1979),

En 1998 Sánchez realizo su estudió "El exceso de información publicitaria" de resultados similares a sus antecesores, pero afirmando que cualquier estudio posterior debía hacer una división entre un clutter competitivo y un no competitivo, dicho de otra manera, saturación publicitaria en la que existe una alta concentración de anuncios en la misma categoría del bien o servicio y los no competitivos, en los que si existe saturación publicitaria pero no de anuncios en la misma categoría de productos. Sánchez (1998)

En Australia entre el año 2001 y el 2007, se llevó a cabo una investigación de carácter empírico sobre las implicaciones de exponer al público a una cantidad excesiva de mensajes publicitarios escrito por Hammer, Riebe y Kennedy (2009)

Los autores centraron su atención en los medios de comunicación preponderantes en aquel entonces; radio y televisión.
Para su metodología, los autores pusieron a prueba la televisión y la radio en dos ambientes completamente diferentes, un ambiente controlado y un entorno real. Durante la misma, se utiliza el término "Clutter" para referirse a la saturación publicitaria.

Ambiente mediado

Radio: 241 participantes escucharon un programa de 20 minutos en un laboratorio. Los participantes se expusieron a un clutter alto ( 9 anuncios) 0 bajo ( 3 anuncios).

Tele: 430 participantes vieron un programa de 1 hora en sus casas. Los participantes se expusieron a un clutter alto (36 anuncios) o bajo (18 anuncios).

Mundo real

Radio: 2650 llamadas a radioescuchas de las emisoras más grandes, incluyendo dos emisoras con poco clutter. Los participantes se expusieron a un clutter bajo (10 anuncios) 0 alto (11 anuncios o más) por hora y media de programación.

Tele: 183 llamadas a televidentes. Los participantes se expusieron a un clutter bajo (37 a 39 anuncios) 0 alto (46 a 48 anuncios) por hora.

Cabe resaltar que los autores reconocen la subjetividad de algunos rangos, sobre todo los que pertenecen al ambiente del mundo real y en cuanto a determinar que representa un clutter alto y uno bajo. Hammer, Riebe y Kennedy (2009)

Al finalizar el proyecto, los autores llegaron a las siguientes conclusiones:

-Cuando hay más publicidad, es decir, un clutter más alto, las audiencias ven y escuchan más comerciales.

-Las audiencias recuerdan una mayor porción de los anuncios a los que se exponen cuando ven o escuchan menos anuncios.

-Las audiencias no están mejor capacitadas para identificar la marca correctamente cuando hay menos ruido en la publicidad.

-Los anuncios que se recordaron de los grupos con exposición a un clutter más alto, simpatizaban más a la audiencia.

- Las investigaciones futuras deberían enfocarse en establecer condiciones de enlace, que pueden relacionarse con la variación de efectos en los diferentes tipos de programas, publicidad para diferentes categorías o para diferentes segmentos de mercado. Hammer, Riebe y Kennedy (2009)

Como adicional, algunos autores proponen algunas romper la barrera de la indiferencia causada por la saturación publicitaria por medio de un énfasis en el contenido de los anuncios, la obra de Thomas Davenport y John Beck conocida como "The attention 
economy" nos dice que el público presenta 6 niveles distintos de atención que podrían ayudar a combatir la saturación. Los cuales son:

- Atención negativa: Esta es causada por el factor shock o por elementos desagradables a los que naturalmente reaccionamos con un estado de alerta, esta publicidad puede incluir actores de apariencia desagradable, alusiones al peligro, muerte o enfermedad, malos sentimientos como la derrota o la agonía, etc.

- Atención positiva: aquí interviene lo contrario a la atención negativa ya que esta publicidad evoca aspiraciones positivas de la vida como: personas guapas, sentimientos buenos, superación personal, victoria, felicidad, etc.

- Atención cautiva: generada por situaciones en las que el público se ve obligado a consumir publicidad debido a circunstancias preparadas. Como ejemplo tenemos la publicidad antes de una película en los cines, el intermedio de un evento deportivos, asignaciones de trabajo, mal tiempo, etc.

- Atención voluntaria: Generada en actividades a las que el público se somete voluntariamente como en un pasatiempo, publicidad gráfica, un arcoíris, etc.

- Atención inconsciente: Publicidad enviada de manera furtiva o subliminal, el usuario no está consciente que se encuentra expuesto a la publicidad, en particular, la publicidad subliminal se considera ilegal en muchos países por lo que ha caído en desuso al ser un método poco ético que afecta el subconsciente del público, manipulando comportamientos con técnicas psicológicas.

- Atención consciente: similar a la atención voluntaria, el consumidor busca la publicidad para informarse antes de una decisión de compra o para comparar determinados bienes o servicios.

Resulta lamentable que dichos niveles de atención se encuentran sobrepasados por el alto nivel de saturación publicitaria al que nos encontramos expuestos en nuestro diario vivir. En el mismo estudio, se nos presentan dos maneras adicionales de combatir los efectos de la saturación publicitaria en caso de que los primeros 6 niveles de atención no tengan efecto.

1) Una exposición mayor al promedio: la estrategia consiste básicamente en aumentar el número, tiempo y tamaño de los anuncios de manera que se pueda obtener una cobertura más amplia y por probabilidad lograr captar la atención del cliente. Como se puede inferir, aumentar la cobertura de una estrategia publicitaria conlleva un incremento en los costos y un aumento en el presupuesto destinado a publicidad, situación que puede no ser redituable y que por lo tanto no justifica la inversión.

2) Innovación: básicamente realizar una campaña arriesgada que rompa paradigmas y que por consiguiente llame la atención, esta alternativa sugiere hacer cosas inesperadas 0 poco habituales que atraigan la atención por la frescura de la publicidad. Si bien innovar es el ideal buscado por muchas empresas, implica muchos desafíos ya que cualquier innovación presenta riesgos e incertidumbre para la marca. Davenport y Beck, (2002).

\section{METODOLOGÍA}

El artículo cuenta con un enfoque exploratorio y

Para conocer cuál ha sido el impacto de la saturación publicitaria en los medios digitales, se hará uso la investigación documental por medio de técnicas cualitativas, se pretende llevar a cabo la revisión de diversas fuentes bibliográficas como: artículos, libros, ensayos, tesis, etc.

La presente investigación contara con tres fases perfectamente definidas para su implementación:

- Fase investigadora: Se procede a indagar entre las diferentes fuentes bibliográficas, el objetivo de esta fase es la comparación de la información que ya se conoce con la nueva información, de manera que se establezca una relación entre ambas.

- Fase sistematizadora: se analizará de manera reflexiva la información recopilada para corroborar su validez.

- Fase Expositiva: se ordena el conocimiento adquirido, en esta fase se crea el discurso científico para exponer la información recopilada de manera clara y precisa.

Para la recolección de información se utilizarán ficheros bibliográficos, en los cuales se anotan las ideas más importantes de la investigación, para esto se requiere una lectura minuciosa de la bibliografía recolectada e implica el posterior análisis e 
Roy Priego Reyes - Germán Martínez Prats - Mariela Adriana Rodríguez Ocaña

interpretación de los datos. Las fichas bibliográficas nos permitirán clasificar la información facilitando su consulta antes de la redacción final.

Finalmente, podemos mencionar el artículo How Clutter Affects Advertising Effectiveness, escrito por Peter Hammer, Erica Riebe y Rachel Kennedy, todo un referente respecto a la saturación publicitaria y que, a pesar de los años, sigue vigente al tratar ampliamente los efectos de este fenómeno en la recordación de marca. La investigación realizada en Australia en el año 2007, sirvió como base para afirmar que existe una relación entre la cantidad de publicidad y el porcentaje de anuncios. A una mayor cantidad de anuncios en el medio, menor es el grado de recordación y viceversa. Hammer, Riebe y Kennedy (2009).

Tabla 1.

Base empírica sobre la saturación publicitaría

\begin{tabular}{|l|l|}
\hline $\begin{array}{l}\text { Webb y Ray } \\
\text { (1979) }\end{array}$ & $\begin{array}{l}\text { Proponen que con una mayor } \\
\text { saturación de publicidad hay } \\
\text { menor porcentaje de recordación, } \\
\text { tanto de anuncios como de marca. } \\
\text { Además, afirman que la audiencia } \\
\text { responde mejor a determinados } \\
\text { tipos de anuncios. }\end{array}$ \\
\hline Cobb (1985) & $\begin{array}{l}\text { La efectividad de la publicidad } \\
\text { disminuye debido a la saturación, } \\
\text { este problema afecta } \\
\text { especialmente a productos y } \\
\text { marcas nuevas. }\end{array}$ \\
\hline Mord y Gilson \\
(1985) & $\begin{array}{l}\text { No hay diferencia en la } \\
\text { recordación de marca cuando hay } \\
\text { saturación publicitaria, sin } \\
\text { embargo, este fenómeno genera } \\
\text { irritabilidad y confusión en la } \\
\text { audiencia. }\end{array}$ \\
\hline Pillai (1990) & $\begin{array}{l}\text { La cantidad de publicidad en el } \\
\text { medio no se impacta de manera } \\
\text { negativa en la audiencia, por lo } \\
\text { tanto, la saturación no genera } \\
\text { rechazo. }\end{array}$ \\
\hline Zhao (1997 & $\begin{array}{l}\text { No existen diferencias } \\
\text { significativas en la recordación } \\
\text { frente a un entorno de saturación } \\
\text { publicitaria. }\end{array}$ \\
\hline Hathschild (1996) & $\begin{array}{l}\text { La audiencia experimenta una } \\
\text { mala actitud ante los anuncios en } \\
\text { exceso. niveles de recordación en } \\
\text { marca y anuncios a mayores } \\
\text { niveles de saturación publicitaria. }\end{array}$ \\
\hline
\end{tabular}

\begin{tabular}{|l|l|}
\hline Dawes (2006) & $\begin{array}{l}\text { Bajos niveles de recordación de } \\
\text { anuncios entre la audiencia debido } \\
\text { a la saturación publicitarían. }\end{array}$ \\
\hline $\begin{array}{l}\text { Hammer, } \\
\text { Riebe y }\end{array}$ & $\begin{array}{l}\text { A una mayor cantidad de anuncios } \\
\text { existe un menor porcentaje de } \\
(2009)\end{array}$ \\
$\begin{array}{l}\text { recordación de anuncios, sin } \\
\text { embargo, la recordación de marca } \\
\text { no se ve afectada. La audiencia } \\
\text { mira y escucha más anuncios sin } \\
\text { rechazo aparente. }\end{array}$ \\
\hline
\end{tabular}

Fuente: Autoría propia basado en Webb y Ray (1979), Cobb (1985), Mord (1985), Pillai (1990), Rothschild (1993), Ha (1996), Zhao (1997, Dawes (2006), Hammer, Riebe y Kennedy (2009).

\section{RESULTADOS:}

\section{Percepción sobre publicidad en internet}

Una gran cantidad de los encuestados deja de visitar un sitio web si percibe que la publicidad en el mismo es excesiva. De igual forma, se opina que la publicidad en internet resulta particularmente más molesta que en otro medio de comunicación como la televisión o la radio. Además, más de la mita considera que se sienten perseguidos por los anuncios de internet.

Tabla 2.

Percepción sobre publicidad en internet.

\section{Opiniones de la audiencia sobre publicidad.}

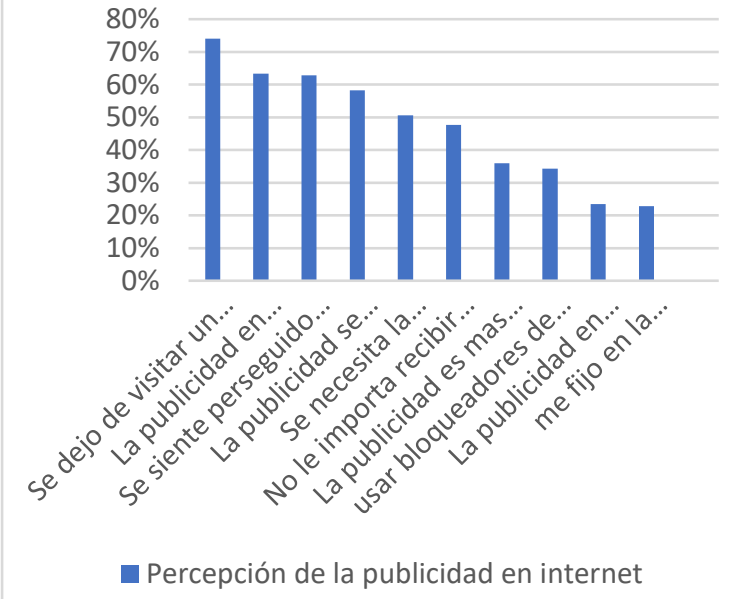

Fuente: Tomado de Infografía Resumen $21^{\circ}$ Navegantes en la Red. Copyright AIMC 


\section{Impacto de la saturación publicitaria en la efectividad publicitaria}

Los autores Hammer, Riebe y Kennedy (2009) demostraron que existe una relación entre la cantidad de publicidad y el porcentaje de recordación de marca y anuncios. A una mayor cantidad de anuncios en el medio, menor es el grado de recordación y viceversa.

Tabla 3.

Impacto de la saturación publicitaria en la memoria (porcentaje de publicidad recordada)

\begin{tabular}{|l|l|l|l|l|}
\hline & $\begin{array}{l}\text { Tiempo } \\
\text { de } \\
\text { exposic } \\
\text { ión }\end{array}$ & $\begin{array}{l}\text { Bajos } \\
\text { niveles } \\
\text { de } \\
\text { publici } \\
\text { dad }\end{array}$ & $\begin{array}{l}\text { Saturac } \\
\text { ión } \\
\text { publicit } \\
\text { aria }\end{array}$ & $\begin{array}{l}\text { Prome } \\
\text { dio }\end{array}$ \\
\hline $\begin{array}{l}\text { Ambiente } \\
\text { experime } \\
\text { ntal }\end{array}$ & $\begin{array}{l}30 \\
\text { minutos }\end{array}$ & 16 & 11 & 13.5 \\
\cline { 2 - 5 } & 1 hora & 45 & 42 & 43.5 \\
\hline $\begin{array}{l}\text { Ambiente } \\
\text { real }\end{array}$ & $\begin{array}{l}\text { Indefini } \\
\text { do }\end{array}$ & 47 & 39 & 43 \\
\hline Promedio & & 36 & 30.6 & \\
\hline
\end{tabular}

Fuente: Hammer, Riebe y Kennedy (2009)

Como se observa en la tabla 1. La audiencia recuerda una mayor proporción de anuncios cuando los niveles de publicidad son más bajos, por otro lado, en un ambiente de saturación publicitaria la recordación disminuye, lo que significa que hay anuncios que no son recordados por la audiencia y, por lo tanto, la efectividad de un porcentaje significativo de anuncios se ve afectada de manera negativa al no poder comunicar correctamente su mensaje.

Tabla 4.

Impacto de la saturación publicitaria en la identificación de marca. (porcentaje de publicidad recordada con la correcta identificación de marca)

\begin{tabular}{|l|l|l|l|l|}
\hline & $\begin{array}{l}\text { Tiempo } \\
\text { de } \\
\text { exposic } \\
\text { ión }\end{array}$ & $\begin{array}{l}\text { Bajos } \\
\text { niveles } \\
\text { de } \\
\text { publici } \\
\text { dad }\end{array}$ & $\begin{array}{l}\text { Saturac } \\
\text { ión } \\
\text { publicit } \\
\text { aria }\end{array}$ & $\begin{array}{l}\text { Prome } \\
\text { dio }\end{array}$ \\
\hline $\begin{array}{l}30 \\
\text { minutos }\end{array}$ & 20 & 30 & 25 \\
\hline
\end{tabular}

\begin{tabular}{|l|l|l|l|l|}
\hline $\begin{array}{l}\text { Ambiente } \\
\text { experime } \\
\text { ntal }\end{array}$ & 1 hora & 58 & 68 & 63 \\
\hline $\begin{array}{l}\text { Ambiente } \\
\text { real }\end{array}$ & $\begin{array}{l}\text { Indefini } \\
\text { do }\end{array}$ & 22 & 39 & 30.5 \\
\hline Promedio & & 33.3 & 45.6 & \\
\hline
\end{tabular}

Fuente: Hammer, Riebe y Kennedy (2009)

Contrario a lo que la revisión de literatura afirma, la saturación publicitaria no afecto de manera negativa a la identificación de marca, por el contrario, altos niveles de publicidad permitieron un mayor porcentaje en la recordación de estas.

\section{DISCUSIÓN Y CONCLUSIONES}

Es evidente que la saturación publicitaria representa un problema para las empresas y el público en general. La investigación determina que la audiencia reacciona de manera desfavorable ante este fenómeno. Reacciones como evitar una página por publicidad excesiva, van totalmente en contra de cualquier objetivo mercadológico. Las encuestas anteriormente analizadas, demuestran que la mayor parte de los internautas tiende a asociar la saturación con sentimientos desagradables y la perciben como una molestia que es mejor evitar. Eguizábal (2008)

Por otro lado, Hammer, Riebe y Kennedy (2009) agregan que mientras aumenta el número de anuncios publicitarios, la recordación de estos disminuye significativamente, sin embargo, los resultados también demuestran que la recordación de marcas no se ve perjudicada por este problema. Por lo anterior se infiere que la saturación si perjudica la recordación de la audiencia, al mismo tiempo que lo impulsa a evitar los anuncios, pero no así a la recordación de una marca.

\section{REFERENCIAS:}

AIMC. (06 de Marzo de 2019). Obtenido de https://www.aimc.es/otros-estudiostrabajos/navegantes-la-red/infografiaresumen-21o-navegantes-la-red/

Brown y Rottshield (1979), Reassessing the Impact of Television Advertising Clutter. Chicago Journal. 
Cobb, C.J. (1985), "Television Clutter and Advertising Effectiveness", in Proceedings of the 1985 AMA Educators' Conference. Chicago: American Marketing Association.

Davenport y Beck, (2002). The attention economy $\left(1^{\circ}\right.$ ed., pp. 78-79). Estados Unidos

Dirconfidencial. (01 de Diciembre de 2015). Obtenido de

https://dircomfidencial.com/marketing/548120151201-0200/

Eguizábal. (2018, 05). eMarketer Releases New Global Media Ad Spending Estimates. Emarketer. Obtenido 03, 2019, de https://www.emarketer.com/content/emarkete r-total-media-ad-spending-worldwide-willrise-7-4-in-2018

Eguizábal, R. (2008). Teoría general de la Publicidad. España: Cátedra.

Galimberti, Cés y González (2007), Patrones de consumo en crecimiento: Repercusiones de la explosión multimedia en las variables de consumo publicitario (pp.145-189). Buenos Aires.

Ha, Louise (1996), "Observations: Advertising Clutter in Consumer Magazines:

Dimensions and Effects", Journal of Advertising Research.

Hammer, P., Riebe, E., \& Kennedy, R. (2009). How Clutter Affects Advertising Effectiveness. Journal of Advertising Research, 49(2), 159163.

https://doi.org/10.2501/s0021849909090217

Mord y Gilson (1985), The Effects of Length, Content, and Repetition on Television. Sánchez (1998), El exceso de información publicitaria. Madrid.

Noguez , O. $(2016,09)$. ¿Qué es un consumidor digital?. Merca 2.0 . Obtenido 11, 2018, de https://www.merca20.com/que-es-unconsumidor-digital/
Pillai (1990). Impact of clutter on advertising viewership and recall: An Indian experiment. Journal of the market Research Society. 187196

Ritacco, E. (29 de agosto de 2016). Adlatina. Obtenido de http://www.adlatina.com/publicidad/lasaturación-publicitaria-el-gran-enemigo-dela-publicidad

Romero, J. (Diciembre de 2015). El funcionamiento del Eye Tracking para el Marketing. Obtenido de Neuromarketing.la :

https://neuromarketing.la/2015/10/elfuncionamiento-del-eye-tracking-para-elmarketing/

Sánchez (1998), El exceso de información publicitaria. (pp. 34-48) Madrid.

Webb, P.H. M Ray (1979), "Effects of television clutter", Journal of Advertising Research, (pp.19, 7 - 12.)

Zhao, X. (1997), "Clutter and Serial Order Redefined and Retested", Journal of

Advertising Research. 